\title{
Study of the Magnetic Properties of Ni/Ag Superlattice
}

\author{
R. Masrour • M. Hamedoun • A. Benyoussef • H. Lassri
}

Received: 9 November 2009 / Accepted: 16 December 2009 / Published online: 7 January 2010

(C) The Author(s) 2010. This article is published with open access at Springerlink.com

\begin{abstract}
Thin multilayer films of alternating ultrathin $\mathrm{Ni}$ and Ag layers $(L(\mathrm{Ni})=11,15,30 \AA$, bulk and $L(\mathrm{Ag})=$ $50 \AA$ ) have been prepared by evaporation in ultrahigh vacuum under controlled conditions and have been studied by the magnetic measurements. The critical temperature $T_{\mathrm{C}}$ is studied as a function of the surface exchange interaction $\left(J_{\mathrm{S}}\right)$. The dependence of $T_{\mathrm{C}}$ on the thickness $L$ of the film has been investigated. A critical value of the surface exchange interaction in the film, above which the surface magnetism appears, is obtained. The shift of the critical temperature $T_{\mathrm{C}}(L)$ from the bulk value $\left[\frac{T_{\mathrm{C}}(\infty)}{T_{\mathrm{C}}(L)}-1\right]$ can be described by a power law $L^{-\lambda}$, where $\lambda=\frac{1}{v_{\mathrm{b}}}$ is the inverse of the correlation length's exponent. The effective critical exponent associated with the magnetization $M(\beta)$ is deduced for different thicknesses of Ni layers, and the thickness $L(\mathrm{Ag})$ was being kept constant at $50 \AA$.
\end{abstract}

\footnotetext{
R. Masrour ( $\square)$

Laboratoire de Physique du Solide, Faculté des Sciences,

Université Sidi Mohammed Ben Abdellah, B.P. 1796, Fes,

Morocco

e-mail: rachidmasrour@hotmail.com

R. Masrour · A. Benyoussef

LMPHE, Faculté des Sciences, Université Mohamed V, Rabat, Morocco
}

M. Hamedoun · A. Benyoussef

Académie Hassan II des Sciences et Techniques, Rabat, Morocco

M. Hamedoun · A. Benyoussef

Institute for Nanomaterials and Nanotechnologies, Rabat,

Morocco

H. Lassri

Département de Physique, Faculté des Sciences Ain Chock, Université Hassan II, Mâarif Route d'El Jadida, km-8, B.P. 5366, Casablanca, Morocco
Keywords Multilayer films · Critical temperature . Exchange interaction $\cdot$ Effective critical exponents

\section{Introduction}

The magnetic properties of magnetic thin film and multilayer systems have been the subject of intense research in recent years [1-13]. With the advance of modern vacuum sciences and in particular molecular beam epitaxy technology, it is now possible to grow magnetic films with few atomic layers or even monolayer atop nonmagnetic substrates in a very controlled way [14-17]. The size in the lateral directions is of infinite extent in thin layers, but restricted by the layer thickness in the third direction. Since the correlation length in the third direction is terminated by the layer thickness, thin layers are ideal media for the studies of finite-size effects on the critical behavior. The critical reduced temperature of the system, $\tau_{\mathrm{C}}$, is studied as a function of thickness of the film and the exchange interactions in the bulk, and within the surfaces $J_{\mathrm{b}}, J_{\mathrm{S}}$ and $J_{\mathrm{b}}$, respectively [18]. A critical value of surface exchange interaction above which surface magnetism appears is obtained. The dependence of the reduced critical temperature on the film thickness $L$ has been investigated [18]. The magnetic properties of multilayer are strongly dependent on its detailed structure and composition, and are determined by the growth conditions used during fabrication [19-21]. For example, a degree of mixing between adjacent layers determines the amount of $\mathrm{Ni}$ needed to contribute to the magnetic properties of the film, and the degree of crystallographic texture within the layers, combined with any surface anisotropy present, determines the overall anisotropy of the multilayer. Since Ni is ferromagnetic, a study of the magnetic properties of this system can also bring additional information on the state of 


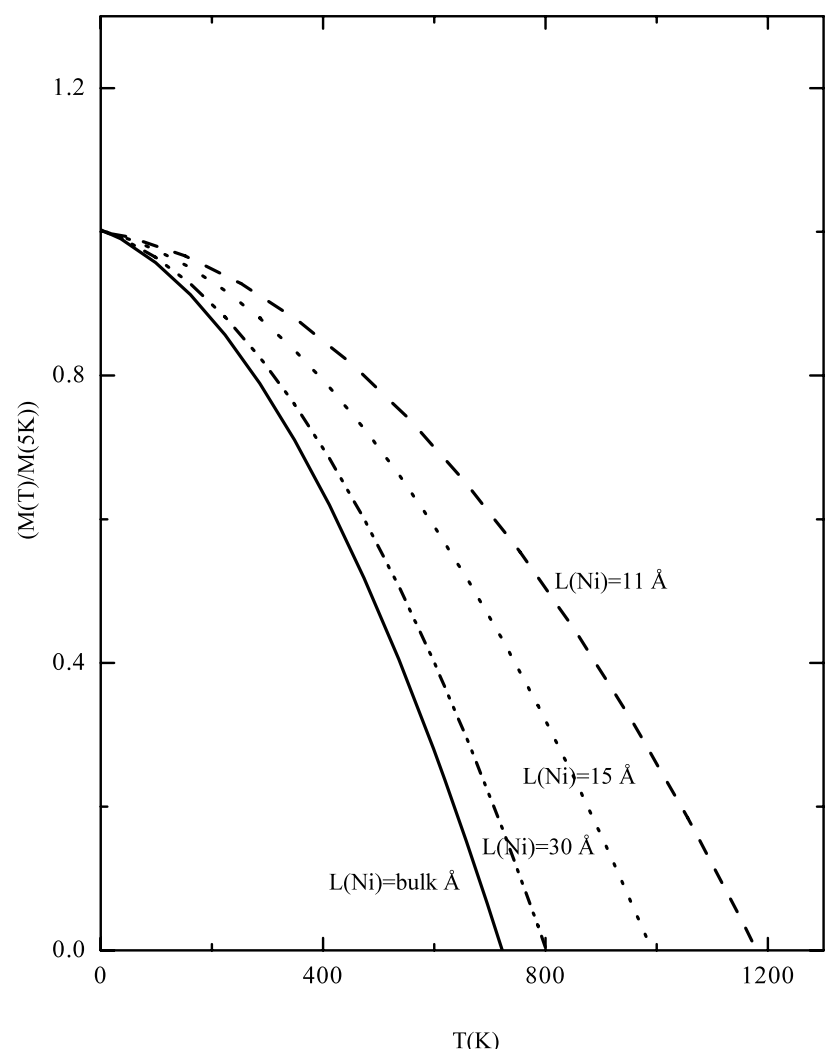

Fig. 1 The temperature dependence of the normalized magnetization of Ni/Ag multilayer with varying thickness of Ni layer

the interface. Therefore, we have undertaken such a study and describe our results here. In this work, the thermal variation of the magnetization as a function of Ni layer thickness is given for different thicknesses of Ni layers and in the range temperature $(0 \leq T \leq 1200 \mathrm{~K})$. The critical temperature and the surface exchange interaction $\left(J_{\mathrm{S}}\right)$ are given for the different thicknesses. The effective critical exponent associated with the correlation length and with the magnetization are obtained for different thicknesses of Ni layers, and the thickness $L(\mathrm{Ag})$ was being kept constant at $50 \AA$.

\section{Experimental}

$\mathrm{Ni} / \mathrm{Ag}$ multilayered films were synthesized by sequential evaporation of $\mathrm{Ni}$ and $\mathrm{Ag}$ in an ultrahigh vacuum using several electron guns. The pressure during evaporation was of the $5 \times 10^{-9}$ Torr order. The rate of evaporation and the thickness were controlled by quartz oscillators. Water cooled glass and Si substrates were used. In all the cases the first layer was $\mathrm{Ag}$. The total number of layers $(N)$ was adjusted to get a total Ni layer thickness of about $1000 \AA$. The Ag layer thickness $L(\mathrm{Ag})$ was being kept constant at $50 \AA$ and $L(\mathrm{Ni})$ varied between 10 and $100 \AA$. Low-angle $\mathrm{x}$-ray diffraction of all the samples revealed peaks typical

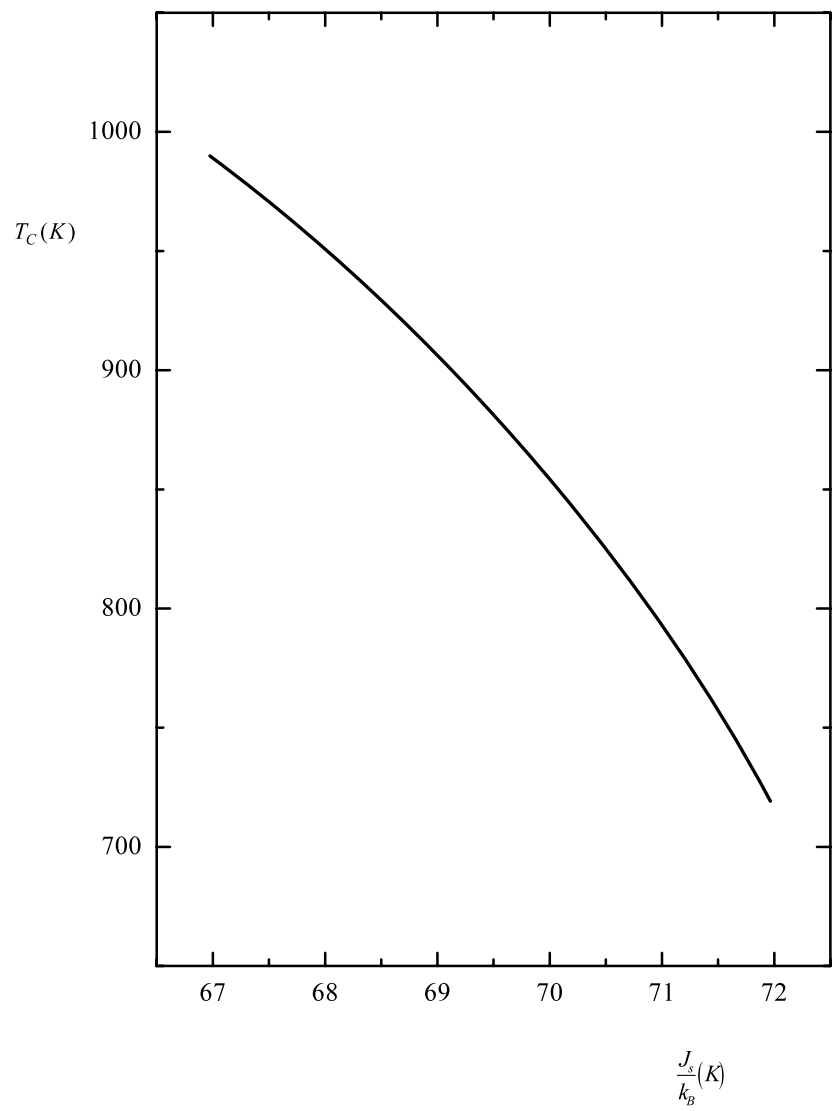

Fig. 2 The critical temperature versus surface exchange interaction $\left(\frac{J_{\mathrm{s}}}{k_{\mathrm{B}}}(K), k_{\mathrm{B}}\right.$ is the Boltzmann's constant) for the Ag layer thickness $L(\mathrm{Ag})$ being kept constant at $50 \AA$ and $L(\mathrm{Ni})$ varied between 10 and $100 \AA$

of the modulated structure, and the x-ray diffraction in the high angle range $35^{\circ}<2 \theta<50^{\circ}$ showed the existence of fcc Ni(111) peak. Magnetization $M$ was measured using a vibrating sample magnetometer under magnetic fields of up to $1 \mathrm{~T}$.

\section{Results}

\subsection{Critical Temperatures and Shift Exponents}

The temperature magnetization was studied in detail for a few samples. Plots of the magnetization versus temperature, for different thicknesses of Ni layers, were made for the $\mathrm{Ni} / \mathrm{Ag}$ multilayer and the critical temperature was obtained by extrapolation of the given curves for different thicknesses of Ni layers (Fig. 1).

The variation of the critical temperature and the surface exchange interaction are plotted in Figs. 2 and 3, respectively, with the different thicknesses of Ni layers, made for the Ni/Ag multilayer (see Fig. 4). The Ag layer thickness $L(\mathrm{Ag})$ was being kept constant at $50 \AA$ and $L(\mathrm{Ni})$ varied 


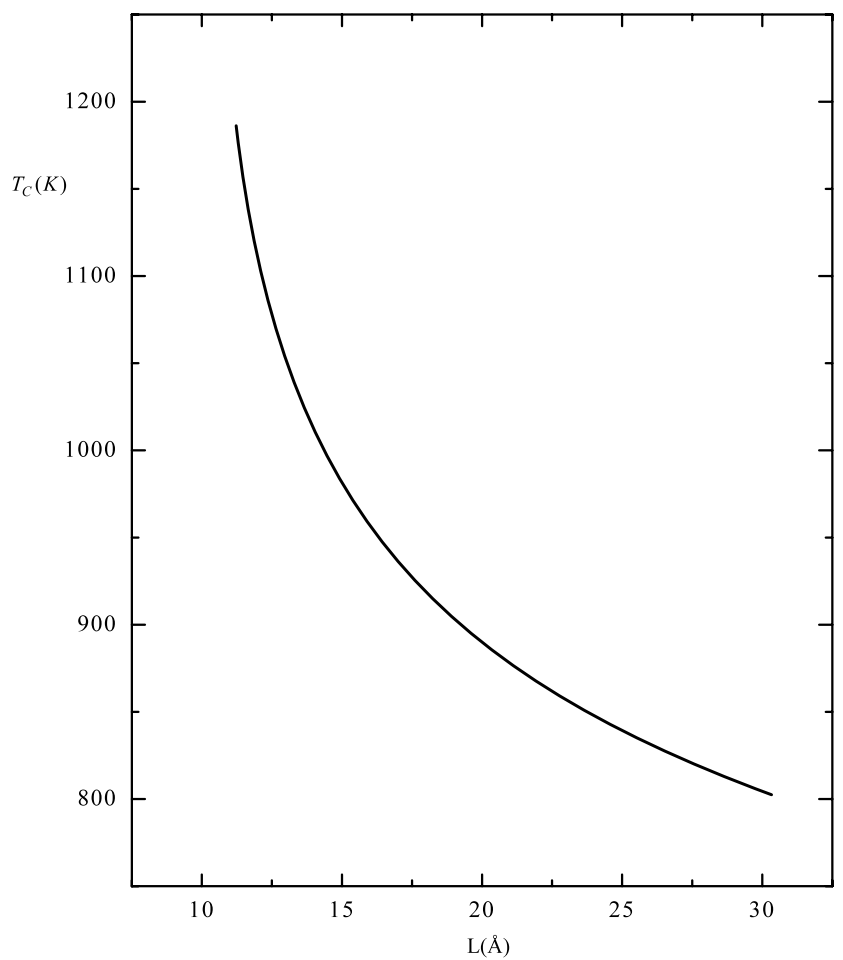

Fig. 3 The critical temperature as a function of layer number $L$ for the Ag layer thickness $L(\mathrm{Ag})$ being kept constant at $50 \AA$ and $L(\mathrm{Ni})$ varied between 10 and bulk $\AA$

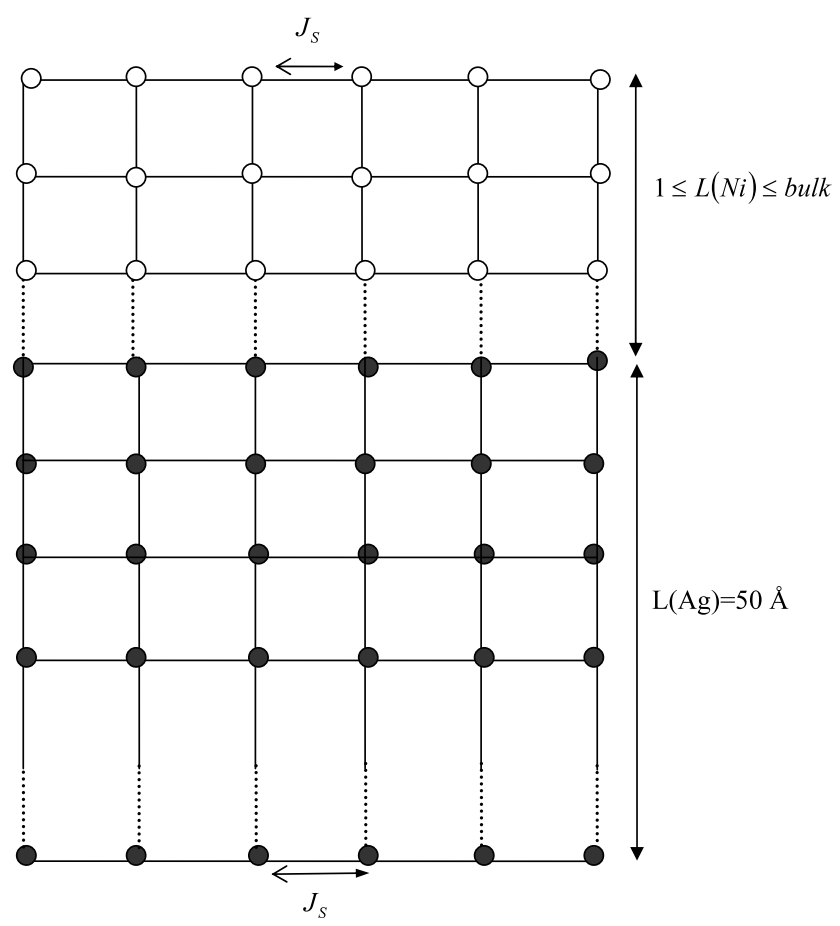

Fig. 4 Two-dimensional cross section of unit cell of infinite superlattice composed of two materials, $\mathrm{Ni}$ and $\mathrm{Ag}$ between 10 and bulk $\AA$. On the other hand, according to the universality hypothesis, critical phenomena can be described by quantities that do not depend on the microscopic details of the system, but only on global properties, such as the dimensionality and the symmetry of the order parameter.

According to the finite-size scaling theory, it has been shown that the approach of $T_{\mathrm{C}}(L)$ to $T_{\mathrm{C}}(\infty)$ can also be described by a simple power law [22] characterized by a shift exponent $\lambda$ defined by:

$\frac{T_{\mathrm{C}}(\infty)}{T_{\mathrm{C}}(L)}-1 \propto L^{-\lambda}$

The shift exponent $\lambda$ is given by $\lambda=\frac{1}{\nu_{\mathrm{b}}}$ where $\nu_{\mathrm{b}}$ is the correlation length's effective critical exponent. The obtained value of $\nu_{\mathrm{b}}$ is obtained as $\left(\nu_{\mathrm{b}}=0.68 \pm 0.03\right)$. This value is between the values given by [23-25], which are

$\nu_{\mathrm{b}}=0.66 \pm 0.03$ and 0.707 ,

respectively. This result substantiates the general universality principle [26].

\subsection{Effective Critical Exponents}

From the result given in Sect. 3.1, we have determined the variation of the $\log \left(M / M_{\mathrm{S}}\right)$ versus $\log \left(1-T / T_{\mathrm{C}}\right)$ for deducing the effective critical exponent $\beta$ given by $M / M_{\mathrm{s}}=$ $\left(1-T / T_{\mathrm{C}}\right)^{-\beta}$, for different thicknesses of Ni layers while the thickness $\mathrm{Li}(\mathrm{Ag})$ was being kept constant at $50 \AA$, where $T_{\mathrm{C}}$ and $M_{\mathrm{S}}$ are the critical temperature and the saturation magnetization (see Fig. 1). The central value obtained is $\beta=0.304 \pm 0.05$ (see Fig. 5).

\section{Discussion and Conclusions}

In summary, the thermal variation of magnetization in multilayer films was calculated and we have deduced the critical temperature and the surface exchange interaction for different thicknesses. The critical temperature decreases with the surface exchange interaction (see Fig. 2) and increases with the number of layers $L(\mathrm{Ni})$ (see Fig. 3). According to the universality hypothesis, critical phenomena can be described by quantities that do not depend on the microscopic details of the system, but only on global properties, such as the dimensionality and the symmetry of the order parameter. The effective critical exponent $v_{\mathrm{b}}$ associated with the magnetic value $\frac{T_{\mathrm{C}}(\infty)}{T_{\mathrm{C}}(L)}-1 \propto L^{-\lambda}$, for different thicknesses of Ni layers and Ag layer of thickness $\mathrm{Li}(\mathrm{Ag})$ being kept constant at $50 \AA$, is calculated. The effective critical exponent $\beta$ associated with the magnetization, for different thicknesses of $\mathrm{Ni}$ layers while the thickness $L(\mathrm{Ag})$ was being kept constant at 


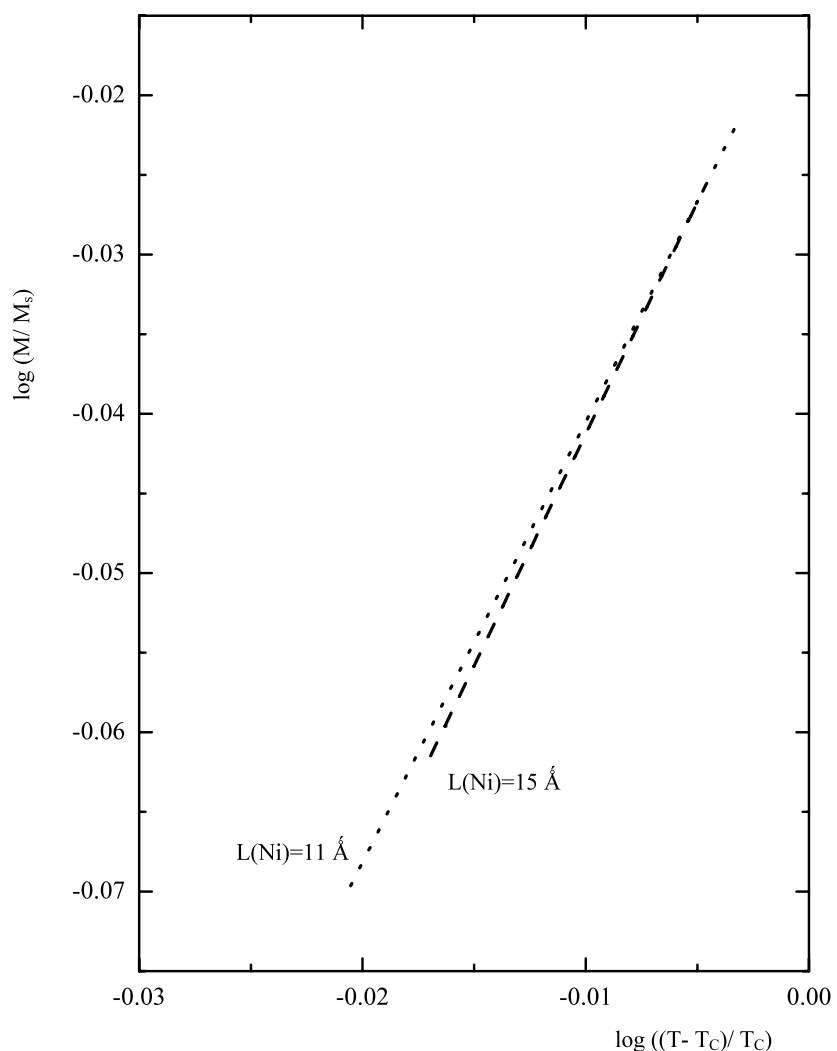

Fig. 5 Determination of the effective critical exponents $\beta$ for the $\mathrm{Ag}$ layer of thickness $L(\mathrm{Ag})$ being kept constant at $50 \AA$ and $L(\mathrm{Ni})=10$ and $15 \AA$

$50 \AA$, is deduced, where $T_{\mathrm{C}}$ and $M_{\mathrm{S}}$ are the critical temperature and the saturation magnetization (see Fig. 1). The obtained results are comparable with those obtained by others models and experimental results. The central value obtained is $\beta=0.304 \pm 0.05$. This value is nearest to the Heisenberg model [27, and references therein].

Open Access This article is distributed under the terms of the Creative Commons Attribution Noncommercial License which permits any noncommercial use, distribution, and reproduction in any medium, provided the original author(s) and source are credited.

\section{References}

1. Elkabil, R., Benkirane, K., Lassri, H., Hamdoun, A., Lassri, M., Krishnan, R.: Moroc. J. Condens. Matter 6, 1 (2005)

2. Camley, R.E., Stamps, R.L.: J. Phys. 5, 3727 (1993)

3. Shaulov, G., Seidov, Y.: J. Magn. Magn. Mater. 140-144, 527 (1995)

4. Balcerzak, T., Tucker, J.W.: J. Magn. Magn. Mater. 140-144, 653 (1995)

5. Hai, T., Li, Z.V., Lin, D.L., George, T.F.: J. Magn. Magn. Mater. 97, 227 (1991)

6. Aguilera-Granaja, F., Maran-Lopez, J.L.: Solid State Commun. 47, 155 (1990)

7. Schneider, C.M., Bressler, P., Schuster, P., Kirschner, J., De Miguel, J.J., Miranda, R.: Phys. Rev. Lett. 64, 1059 (1990)

8. Ramos, C.A., Lederman, D., King, A.R., Jaccarino, V.: Phys. Rev. Lett. 65, 2913 (1990)

9. Ambose, T., Chen, C.L.: Phys. Rev. Lett. 76, 1743 (1996)

10. Binder, K., Evans, R., Landau, D.P., Ferrenberg, A.M.: Phys. Rev. E 53, 5023 (1996)

11. Pappas, D.P., Kamper, K.-P., Hopter, H.: Phys. Rev. Lett. 64, 3179 (1996)

12. Camley, R.E., Li, D.: Phys. Rev. Lett. 84, 4709 (2000)

13. Bakrim, H., Bouslykhane, K., Hamedoun, M., Hourmatallah, A., Benzakour, N.: Surf. Sci. 569, 219 (2004)

14. Onellion, M.F., Fu, C.I., Thomson, M.A., Erskine, J.L., Freeman, A.J.: Phys. Rev. B 33, 7322 (1986)

15. Thomson, M., Erskine, J.L.: Phys. Rev. B 31, 6832 (1985)

16. Farle, M., Baberschke, K.: Phys. Rev. Lett. 58, 511 (1987)

17. Dûrr, W., Taborelli, M., Paul, O., Germar, R., Gudat, W., Pescia, D., Landolt, M.: Phys. Rev. Lett. 62, 206 (1989)

18. Masrour, R., Hamedoun, M., Bouslykhane, K., Hourmatallah, A., Benzakour, N., Benyoussef, A.: Appl. Surf. Sci. 255, 7462-7467 (2009)

19. Grundy, P.J., Babkair, S.S., Ohkoshi, M.: IEEE Trans. Magn. MAG-25, 3626 (1989)

20. Krishnan, R., Lassri, H., Seddat, M., Porte, M., Tessier, M.: Appl. Phys. Lett. 64, 2312 (1994)

21. Abid, M., Ouahman, H., Lassri, H., Khmou, A., Krishnan, R.: J. Magn. Magn. Mater. 202, 335 (1999)

22. Domb, C.: J. Phys. A. 6, 1296 (1973)

23. Chen, K., Ferrenberg, A.M., Landau, D.P.: Phys. Rev. B. 48, 3249 (1993)

24. Cabral Neto, J., Ricardo de Sousa, J., Plascak, J.A.: Phys. Rev. B 66, 064417 (2002)

25. Boccara, N.: Symétries Brisées. Hermann, Paris (1976)

26. Kadanoff, L.P.: Statistical Physics: Static Dynamics and Renormalization. World Scientific, Singapore (2000)

27. Collins, M.F.: Magnetic Critical Scattering. Oxford University Press, London (1989) 